\title{
Design and Characterization of a Mode-Splitting $\Psi$-Junction
}

\author{
G. J. Veldhuis, J. H. Berends, and P. V. Lambeck
}

\begin{abstract}
It will be shown in this paper, that the mode conversion factor (MCF) as defined for Y-junctions, can be profitably applied for the design of three branch junctions for splitting the three lowest-order modes of a channel waveguide. Accordingly, these so-called mode-splitting $\Psi$-junctions were designed for implementation in PECVD SiON-technology. Propagation calculations point to crosstalk levels well below $-20 \mathrm{~dB}$ at $10-20$ mm junction length. The produced $\Psi$-junctions show crosstalk of less than $-17 \mathrm{~dB}$, mainly originating from the nonhomogeneity of the refractive indexes of the SiON layers.
\end{abstract}

\section{INTRODUCTION}

$\mathbf{M}$ ULTIBRANCH junctions like the well-known $\mathrm{Y}$ junction $(1 \rightarrow 2)$ or the less-known $\Psi$-junction (1 $\rightarrow 3$ ), which can spatially separate the modes of a channel waveguide, are micro-optical components of great interest [1], [2]. They can be used for a multitude of functions such as selective mode excitation, mode routing and multiport broad-band wavelength multiplexing and demultiplexing. Also the spatial mode of a semiconductor laser can be controlled with these junctions. By electro-optical modulation of the propagation constants of the different branches of the junction, they could be applied as a multiport optical switch. Furthermore these junctions can be used to improve the power distribution in certain types of multibranch power splitters [3] For all these applications the crosstalk and the device length are the most relevant device parameters.

The mode-splitting property of waveguide junctions is based on two effects, mode localization and adiabatic propagation. An extensive study of these two features in n-branch junctions was done by Thurston et al. [4]. They found that maximizing the difference in propagation constants of the uncoupled branches of a junction, will improve both mode localization and adiabatic propagation and therefore reduce the length of the junction or alternatively reduce the crosstalk at a given junction length. They also found that increasing the lateral refractive index contrast or the wavelength will decrease the crosstalk of a given device. In the case of $\Psi$-junctions, they found that the junction angle should be in the order of milliradians.

In this paper we will show that the MCF as defined for $\mathrm{Y}$-junctions [5] can also be of use in describing the behavior of a certain class of $\Psi$-junctions. A design strategy using the MCF will be introduced resulting in minimal junction length

Manuscript received October 18, 1995; revised March 13, 1996

The authors are with the MESA Research Institute, University of Twente NL-7500 AE Enschede, The Netherlands.

Publisher Item Identifier S 0733-8724(96)05429-1. at a desired crosstalk level. We have realized several modesplitting junctions in SiON-technology using this strategy. These junctions were characterized by analyzing power distributions over the branches, intensity profiles in the multimodal branch and the beat-pattern of light scattered out of that branch. The characterizations show that a $\Psi$-junction with a intermodal crosstalk of less than $-17 \mathrm{~dB}$ has been realized. To the best of our knowledge, this is the first report of a properly working mode-splitting $\Psi$-junction.

\section{DEVICE PRINCIPLES}

The mode-splitting behavior of multibranch junctions [2] is caused by two distinct effects, i.e., mode localization and adiabatic propagation. First of all, if the propagation constants of the uncoupled branches of the junction are different, then for large channel separation the mode shapes of the different system modes and the mode shapes of the uncoupled channel modes will be identical. Therefore the power in each system mode will localize in only one of the branches when the separation between the branches increases (see Fig. 1). This is called "mode localization." Second, when the junction angle is small enough, the structure will change very gradually in the propagation direction and the power transfer between the different system modes will be negligible. This is called "adiabatic propagation." Therefore, due to mode localization and adiabatic propagation, power launched at the beginning of the junction in one of the modes of the multimodal branch, will end up in only one of the branches of the junction. Where the lowest order mode localizes in the branch with the highest uncoupled propagation constant and so on. Essentially, the device is reciprocal, e.g., power launched in one of the branches will end up in one particular mode of the multimodal branch.

Mode-splitting junctions will show crosstalk. Due to the two distinct effects that govern the mode splitting, two different contributions to the crosstalk can also be distinguished. These are propagation crosstalk and localization crosstalk, which should both be small. Propagation crosstalk of a system mode in a $\Psi$-junction, will be defined as the ratio between the sum of the power in the two unwanted system modes and the sum of the total power in the guided system modes at the end of the structure. Among other things, it can be reduced by decreasing the junction angle. The localization crosstalk of a system mode will be defined as the ratio between the sum of its power overlap with the unwanted channel modes and the sum of its power overlap with all three channel modes. It can 

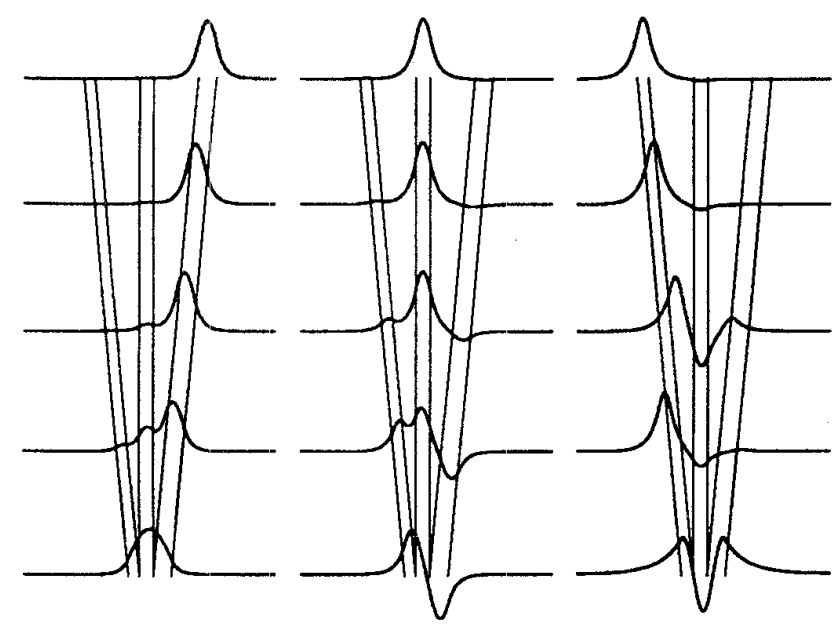

Fig. 1. Typical localization of the modal profile for the system modes of a mode-splitting $\Psi$-junction (top view) for increasing separation between the branches. The branches have different uncoupled propagation constants (see [2, Fig. 1]).

be reduced, for example, by increasing the channel separation at the end of the junction.

\section{APPLICABILITY OF THE MCF TO $\Psi$-JUNCTIONS}

For the well-known Y-junction the quality of the mode splitting can, in a quantitative way, be related to the mode conversion factor (MCF) [5], [6]. It is defined as

$$
\mathrm{MCF}=\frac{\Delta \beta}{\tan \theta \sqrt{\bar{\beta}^{2}-\beta_{b}^{2}}}
$$

where $\theta$ is the angle between the branches, $\Delta \beta$ the difference between the propagation constants of the uncoupled branches, $\bar{\beta}$ the average of these propagation constants and $\beta_{b}=2 \pi n_{b} / \lambda$ with $n_{b}$ the effective refractive index of the background and $\lambda$ the wavelength. For MCF $\ll 0.43$ the junction behaves as a power splitter, but MCF $\gg 0.43$ acts as a mode splitter [5]. Fig. 2 shows a typical example of how the power is distributed over the two modes at the end of a $\mathrm{Y}$-junction as a function of its MCF [6]. It could be said that for MCF $\gg 2$ the propagation crosstalk for a $\mathrm{Y}$-junction can be estimated as less than $-20 \mathrm{~dB}$

When looking at a $\Psi$-junction it is easy to imagine the junction as consisting of three different $\mathrm{Y}$-junctions. Each pair of branches forms a Y-junction for which an MCF can be calculated. It would be tempting to think that the interaction between the two system modes belonging to the branches of each of the sub-junctions (the modes that will localize in these branches), could be described by their MCF. It will be shown that it is sound to do so.

This is qualitatively in line with Thurston's results, because increasing the MCF of a Y-junction corresponds to the parameter variations, which appeared to improve the mode-splitting performance of $n$-branch junctions in general. Therefore, it can be concluded that increasing the MCF's of a given $\Psi$-junction improves its mode-splitting behavior.

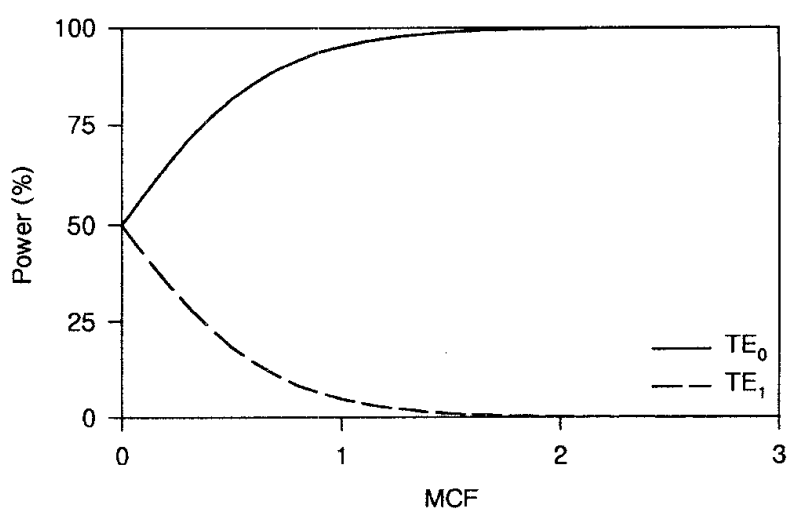

Fig. 2. Typical power distribution over the two system modes after propagation through a Y-junction, as a function of its $\mathrm{MCF}$, upon launching $\mathrm{TE}_{0}$. The solid line shows the power in $\mathrm{TE}_{0}$ and the dotted line the power in $\mathrm{TE}_{1}$.

With this qualitative agreement in mind, its is interesting to investigate if the MCF can be applied for $\Psi$-junctions in a more quantitative way. In this case it is convenient to limit the range of considered junctions to the type which, viewed from above, is depicted in Fig. 3. It consists of a channel waveguide with width $d_{0}$ supporting three guided modes, which splits into three different monomode waveguides with respective widths $d_{1}, d_{2}$, and $d_{3}$ where $d_{0}=d_{1}+d_{2}+d_{3}$. For the calculations, the actual 3-D structure will be reduced to two dimensions using the effective index method [7]. The effective refractive index of the background is $n_{b}$ whereas the effective refractive indexes of the branches is $n_{g}$. The branching angles are $\theta_{1}$ and $\theta_{2}$.

For practical purposes it is inviting to describe the $\Psi$ junction by only one MCF value. This can be arranged in the following way: for a given small lateral refractive index contrast, the square root term in the denominator of (1) is nearly a constant $(C)$, therefore in good approximation the $\mathrm{MCF}$ can be written as

$$
\mathrm{MCF}=\frac{\Delta \beta}{C \cdot \theta} .
$$

If we then require

$$
\begin{aligned}
\theta & =\theta_{1} \\
& =\theta_{2} \\
\beta_{1} & =\beta_{2}-\Delta \beta
\end{aligned}
$$

and

$$
\beta_{3}=\beta_{2}+\Delta \beta
$$

it can easily be seen that the three MCF's of the composing Yjunctions of the $\Psi$-junction are equal, and therefore one single parameter can be attributed to this type of junction.

Beam propagation in the junction has been calculated with the step-approximation method [8], already using the parameters of the SiON-technology-based ridge-waveguide system in which the device will eventually be realized. These are $n_{b}:=1.5400, n_{g}=1.5407$, and the He-Ne wavelength $\lambda=632.8 \mathrm{~nm}$. Since we want to use TM polarized light for the actual device the $2-\mathrm{D}$ structure, resulting from application 


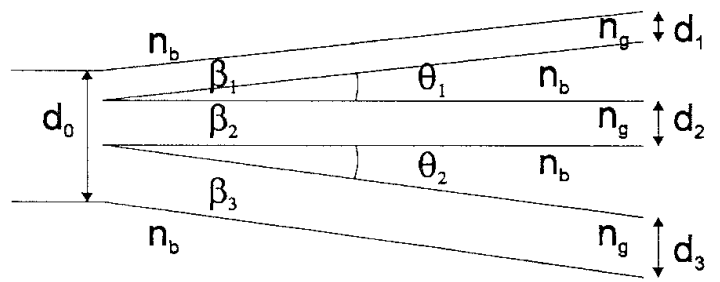

Fig. 3. Top view of a $\Psi$-junction with its relevant parameters.

of the effective index method, has to be worked out for TE polarization.

The mode-splitting performance as a function of the MCF for several Y-junctions is shown in Fig. 4. The curves show the power distribution over the different system modes at the end of the junction $\left(\mathrm{TE}_{n}\right)$ on launching one of the system modes $\left(\mathrm{TE}_{m}\right)$ in the multimode branch. The MCF was varied in two ways. In series A by changing the branching angle, whereas for series B the width of the branches has been varied; all variations obeying (3). The channel separation at the end of all junctions was chosen large enough to assure that the localization crosstalk is less than $-23 \mathrm{~dB}$. This means the device length is sufficient to assure that all of the interaction between the modes of the junction has been taken into account. The figure shows that the variation of the resulting power in the launched mode with the MCF is very similar for all three system modes and also for both simulated series. Furthermore, as expected, there is a great similarity with Fig. 3. It also shows that the mode interaction is dominated by power transfer between $\mathrm{TE}_{0}$ and $\mathrm{TE}_{1}$ and between $\mathrm{TE}_{1}$ and $\mathrm{TE}_{2}$ whereas the interaction between $\mathrm{TE}_{0}$ and $\mathrm{TE}_{2}$ (the two outer branches) is negligible down to $\mathrm{MCF}=1$. The most important conclusion is that the crosstalk between all the system modes is $<-20$ $\mathrm{dB}$ for $\mathrm{MCF}>2.4$.

Although we severely restricted the group of investigated junctions by limiting the number of considered parameters, numerous other simulations indicate that other device configurations with, for example, a different channel order, will not lead to significantly shorter junctions for similar crosstalk performance.

At this point, it is important to remark that, although the calculations were performed for a specific set of physical parameters, for weakly guiding structures $\left\{\left(n_{g}-n_{b}\right) / n b \ll\right.$ $1\}$, the results can be transferred to any refractive index contrast and any wavelength using the device equivalence concept [9]. It can be shown (Appendix A) that equivalent junctions have the same MCF.

It can be concluded that for the given type of $\Psi$-junction, the MCF can be well used for describing the mode-splitting behavior and that for obtaining crosstalk levels $<-20 \mathrm{~dB}$ the MCF should be larger than 2.4.

\section{DEVICE DESIGN AND FABRICATION}

Several $\Psi$-junctions have been realized using PECVD SiON technology [10]. This technology was chosen because the refractive index is easily tunable by varying the $\mathrm{O} / \mathrm{N}$ ratio in the material and for its compatibility with IC technology. In particular, in the refractive index region around $n=1.54$ the

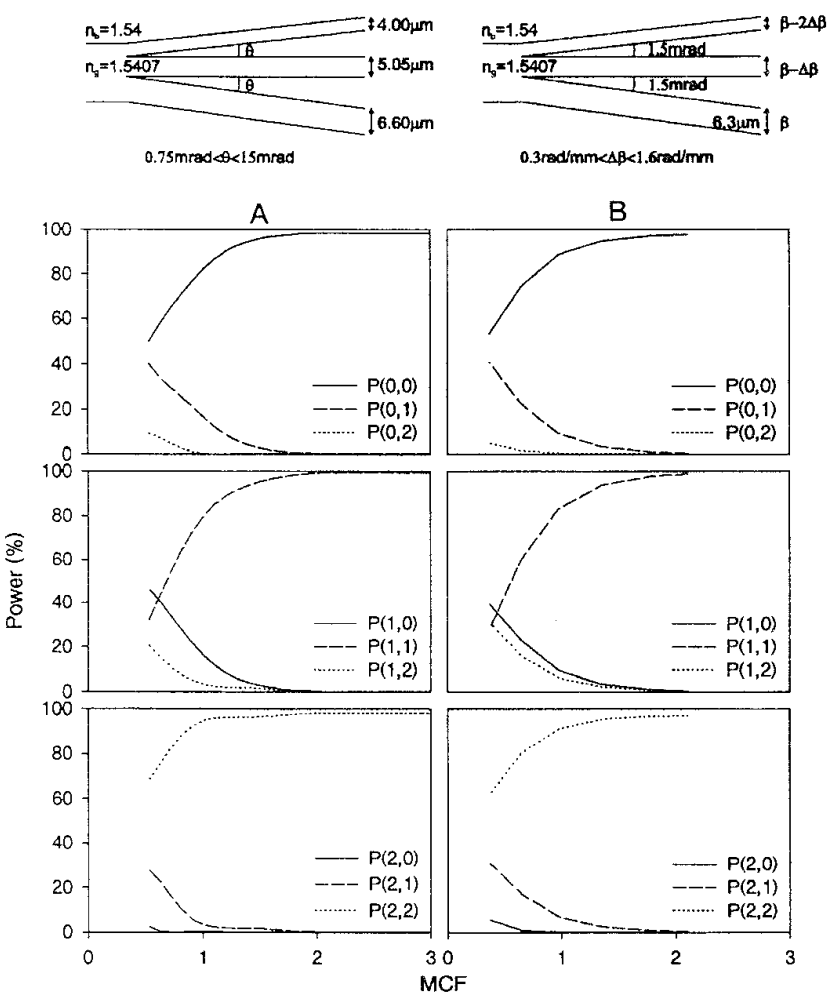

Fig. 4. $P(m, n)$ is the power in mode $\mathrm{TE}_{n}$, at the end of the junction, resulting from launching $\mathrm{TE}_{m}$ in the multi modal branch, shown as a function of MCF. In series A the MCF was varied by changing the branching angle for fixed branch widths. In series B the angle was fixed and the widths of the branches were varied.

homogeneity of the refractive index as well as the uniformity of the layer thickness can be very well controlled. Therefore this type of SiON will be used. In order to couple light into the waveguide efficiently and to facilitate device characterization, we used a small refractive index difference $(0.1)$ between the buffer and the guiding layer, resulting in a thick waveguide. In Fig. 5 the ridge waveguide system is shown. It consists of two SiON layers, PECVD deposited on a Si-wafer. The upper (guiding) SiON layer has a slightly higher refractive index than the bottom (buffer) layer. Their refractive indexes and thicknesses were measured on reference samples, which were simultaneously produced on thermally oxidized silicon wafers, using prism coupling [11]. In the case of the refractive indexes, the values $n_{\text {buffer }}=1.538$ and $n_{\text {guide }}=1.547$ were found, whereas the thicknesses were found to be $t_{\mathrm{b}}=3.70 \mu \mathrm{m}$ and $t_{\mathrm{g}}=2.24 \mu \mathrm{m}$. The nonuniformity of the indexes and the thickness over the wafer were measured and found to be $\pm 0.1 \%$, and $\pm 1 \%$, respectively. A small lateral effective refractive index contrast is necessary as this will leave some room to vary the difference in channel widths of the branches. The channels were defined by reactive ion etching of a $255 \mathrm{~nm}$ deep ridge in the guiding layer, corresponding with a lateral refractive index contrast of approximately $\Delta n=7 \times 10^{-4}$. Then the $\mathrm{TE}_{1}$ cut-off channel width is $6.8 \mu \mathrm{m}$. The etch depth was measured using a stylus surface profiler and a uniformity of $5 \%$ was found. 
TABLE I

Parameters for the Designed $\Psi$-Junction. Here $\Delta n$ is the Lateral Effective Refractive Index Contrast ror Which the Device was Designed

\begin{tabular}{|c|c|c|c|c|c|c|c|}
\hline $\begin{array}{c}\Delta n \\
\left(\times 10^{-4}\right) \\
\end{array}$ & $\begin{array}{c}d_{1} \\
(\mu \mathrm{m})\end{array}$ & $\begin{array}{c}d_{2} \\
(\mu \mathrm{m})\end{array}$ & $\begin{array}{c}d_{3} \\
(\mu \mathrm{m})\end{array}$ & $\begin{array}{c}\theta \\
(\mathrm{mrad})\end{array}$ & $\begin{array}{c}L \\
(\mathrm{~mm})\end{array}$ & MCF & $\begin{array}{c}\mathrm{CT} \\
\text { (dB) }\end{array}$ \\
\hline 4.5 & 4.5 & 5.9 & 8.0 & 1.00 & 20.0 & 2.06 & -23.8 \\
\hline 5.5 & 4.0 & 5.3 & 7.3 & 1.00 & 17.5 & 2.40 & -28.6 \\
\hline 6.5 & 3.5 & 4.8 & 6.8 & 1.22 & 13.5 & 2.41 & -26.4 \\
\hline 7.5 & 3.1 & 4.3 & 6.3 & 1.36 & 11.0 & 2.46 & -24.8 \\
\hline 8.5 & 2.8 & 4.0 & 6.0 & 1.34 & 10.5 & 2.86 & -24.6 \\
\hline 9.5 & 2.7 & 3.8 & 5.7 & 1.32 & 10.0 & 3.02 & -26.6 \\
\hline
\end{tabular}

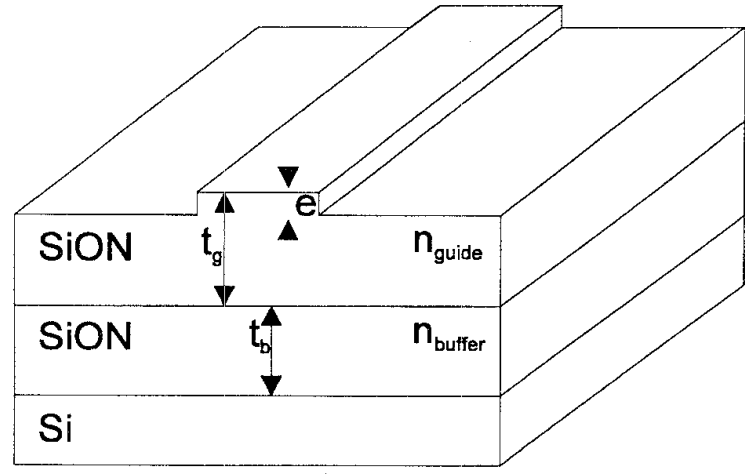

Fig. 5. Ridge waveguides in PECVD SiON technology. The etch depth is $e=255 \mathrm{~nm}, n_{\mathrm{buffer}}=1.538, n_{\mathrm{guide}}=1.547, t_{b}=3.7 \mu \mathrm{m}$ and $t_{i}=2.24 \mu \mathrm{m}$

A worst case analysis with respect to the technological tolerances showed that the nonuniformity in the lateral refractive index contrast can be up to $3 \times 10^{-4}$. This can be mainly attributed to the nonuniformity of the refractive indexes of the two SiON-layers, which, although very small, can be up to $30 \%$ of the vertical index contrast, in a worstcase situation. Since this nonuniformity is too large to assure proper realization of the designed $\Psi$-junction, several devices were designed, each performing optimally for a different value of the lateral effective refractive index contrast. The following procedure has been used for the design.

First, we choose the width of the broadest and the smallest branch and vary the width of the middle branch in order to obtain the same MCF for the two Y-junctions formed by the adjacent branches. It is important to keep in mind that in order to maintain the junction as short as possible, the difference in width between the two outer branches should be chosen as large as possible. Here the width of the broadest branch is limited by the requirement that it is monomodal, but that of the smallest branch either by the requirement that the sum of the branch widths must support three modes, or by the limitations of the photolithography. After this, the junction angle can be calculated using (1) by ensuring MCF $=2.4$. Finally the resulting design was checked by means of a propagation calculation.

Table I shows the parameters of the designed junctions, where $L$ is the device length for which the localization crosstalk equals $-23 \mathrm{~dB}$ and $\mathrm{CT}$ is the propagation crosstalk calculated for the system mode which shows the least adiabatic propagation. The length of the simulated devices was chosen sufficiently large to assure that all of the interaction between the modes of the junction has been taken into account.

\section{Considerations on Device Characterization}

Before realization starts, the question whether characterization is feasible has to be answered. The characterization method that first comes to mind for measuring the performance of a mode-splitting junction, is simply to excite one of the modes of the multimodal branch and then measure the power distribution over the branches at the end of the junction. Unfortunately, due to the small difference in propagation constants, selective excitation of the different channel modes by either end-fire coupling, grating coupling or prism coupling is not possible. With this problem in mind, we have chosen the following methods to characterize the mode-splitting behavior of the fabricated junctions.

Two types of devices were made. First of all, devices consisting of a single $\Psi$-junction as is illustrated in Fig. 6(a). By exciting one of the monomodal branches of the junction a specific channel mode of the multimodal branch is expected to be excited. In practice a small fraction of the inserted power will leak to the other system modes and will result in a distorted near-field intensity distribution in the multimodal branch. This profile can be projected on a CCD camera and the amplitudes and the relative phases of the three modes can then be derived by using the fit-procedure described in Appendix B. Crosstalk will also result in a beat pattern between the channel modes in the outgoing three-modal branch. This beat pattern can be examined by projecting the top-view scatter pattern onto a CCD camera. Since, due to the quadratic relation between the field and the power of a mode, very small values for the crosstalk, e.g., $2 \%$ will already lead to severe beat patterns with intensity variations of $35 \%$, this method gives a good indication of the amount of crosstalk between the different system modes.

The second type of device consists of two identical $\Psi$ junctions mutually connected with the three-modal branch as is illustrated in Fig. 6(b). If one of the branches of the first junction is excited, the power is expected to end up in the same branch of the second junction. However, if the propagation is not completely adiabatic, then there will also be power in the other two branches at the end of the device. The devices are characterized by end-fire excitation of one of the branches and measuring the resulting power distribution. If the device is adiabatic this will be a very strong indication that the two $\Psi$-junctions which make up this device are adiabatic too. Nevertheless there is a small chance that the propagation 


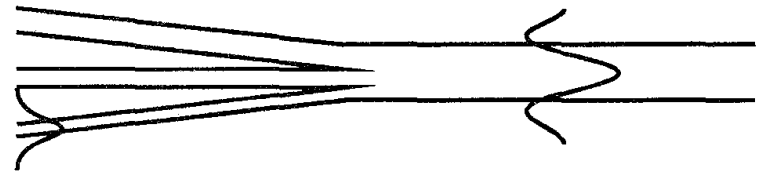

(a)

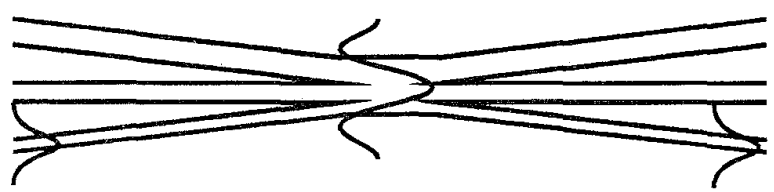

(b)

Fig. 6. (a) Single $\Psi$-junction. Excitation of one of the ingoing branches should result in exciting only one of the channel modes of the outgoing branch. (b) Device consisting of two $\Psi$-junctions. Excitation of one of the ingoing branches should result in power in the same outgoing branch only.

crosstalk for all three modes of the first junction is just cancelled out by the crosstalk of the second junction. In order to confirm this possibility, beat patterns have to be observed in the multimodal branch

In conclusion, for characterization purposes, in addition to single junctions, double structures have also been realized.

\section{MEASUREMENTS AND DisCUSSION}

First, measurements were carried out on the devices consisting of two $\Psi$-junctions. TM polarized light of a $\mathrm{He}-\mathrm{Ne}$ laser was focused on the end face of one of the ingoing branches of the device. The three resulting spots at the end of the device were each projected onto a different detector. The crosstalk was defined as the sum of the power in the unwanted branches divided by the total power in the three branches. Since we are only interested in the propagation crosstalk, the devices were left somewhat longer $(4.5 \mathrm{~cm}$ in total) than the designed devices to assure that no mode-localization effects were included in the measurements.

The results of the measurements are given in Table II. It can be seen that all devices are very adiabatic, the highest crosstalk level was measured and found to be $-16.2 \mathrm{~dB}$ whereas the best value was $-17.2 \mathrm{~dB}$. The junctions each were designed for a different lateral effective refractive index contrast, therefore it is expected that not all of them work properly at the same time. The devices designed for higher refractive index contrast showed no propagation for the $\mathrm{TM}_{2}$ system mode, which means that the middle part of the device is only bi-modal. This, in turn, means that the actual realized lateral effective refractive index contrast $\Delta n<7 \times 10^{-4}$ at the position of the multimodal branches of these devices on the wafer. Although the devices were all designed for a different lateral refractive index contrast there is no large difference in performance between the different devices. This can be attributed to the inhomogeneity in the refractive index of the SiON layers. Since the devices are quite long, the lateral effective refractive index contrast will change on propagation through the device.

The measured mode profiles for a junction designed for $\Delta n=5.5 \times 10^{-4}$ are shown in Fig. 7. Clearly the modal
TABLE II

Propagation Crosstalk Between the System Modes for Devices Consisting of Two $\Psi$-Junctions With the Parameters as Described in Table 1 . Here $\Delta n$ is the Lateral Effective Refractive Index Contrast for which the Device was Designed

\begin{tabular}{cccc}
\hline $\begin{array}{c}\Delta n \\
\left(\times 10^{-4}\right)\end{array}$ & $\begin{array}{c}\mathrm{TM}_{0} \rightarrow \mathrm{TM}_{\mathbf{1 + 2}} \\
(\mathrm{dB})\end{array}$ & $\begin{array}{c}\mathrm{TM}_{1} \rightarrow \mathrm{TM}_{0+2} \\
(\mathrm{~dB})\end{array}$ & $\begin{array}{c}\mathrm{TM}_{2} \rightarrow \mathrm{TM}_{0+1} \\
(\mathrm{~dB})\end{array}$ \\
\hline 4.5 & -18.6 & -17.7 & $\mathbf{- 1 6 . 6}$ \\
5.5 & -18.9 & $-\mathbf{1 7 . 2}$ & -17.7 \\
6.5 & -18.6 & -18.8 & $\mathbf{- 1 6 . 2}$ \\
$7.5-9.5$ & The middle region of the device is only bimodal \\
\hline
\end{tabular}

features of the three lowest-order modes can be recognized. From a least-square fit, as described in Appendix B, to the measured data for the second-order mode (the line in Fig. 7), the values $V=8.9 \pm 0.1$ and $w=16.7 \pm 0.1 \mu \mathrm{m}$ were found for the normalized frequency and the channel width. The power fractions for the different guided modes were calculated to be $0.2 \%, 1.8 \%$, and $98.0 \%$ for $\mathrm{TM}_{0}, \mathrm{TM}_{1}$, and $\mathrm{TM}_{2}$, respectively. Fits on the lower order modes indicated low crosstalk of a few percent, but due to the strong correlation between phase and amplitude of the lower-order modes, the calculated standard deviations of the parameters were too high to give accurate values for this crosstalk. As a check for the validity of the model it can be seen that the channel width resulting from the fit procedure, $16.7 \pm 0.1 \mu \mathrm{m}$, is in excellent agreement with the designed channel width of $16.6 \mu \mathrm{m}$. The effective refractive index contrast at the end of the junction was calculated from the values found for the normalized frequency and the channel width and resulted in $9.3 \times 10^{-4}$. If this contrast were valid along the whole junction, we could expect very poor values for the crosstalk. Also the devices consisting of two junctions would all have been three-modal. Therefore this value is a strong indication that the refractive index contrast is not uniform in the propagation direction. Also the main part of the crosstalk, which is somewhat larger than the crosstalk calculated for the junctions, can be attributed to this nonuniformity.

Finally, the mode profiles of the multimodal branch were examined by looking at the scattered light. Typical examples of measured scatter patterns for exciting the three different branches of the junction can be seen in Fig. 8. Again the modal features of the three lowest order modes can be clearly recognized. Since we have already learned that the crosstalk between $\mathrm{TM}_{0}$ and $\mathrm{TM}_{2}$ and that between $\mathrm{TM}_{1}$ and $\mathrm{TM}_{2}$ is very small, we are mainly interested in the crosstalk between $\mathrm{TM}_{0}$ and $\mathrm{TM}_{1}$. The beat length is about $1.6 \mathrm{~mm}$ and therefore a beat pattern cannot be seen in Fig. 8. By scanning along the waveguide with a microscope the beat pattern can be examined by eye. No beat pattern was observed and therefore it can be concluded that the crosstalk between $\mathrm{TM}_{0}$ and $\mathrm{TM}_{1}$ should be well below $-17 \mathrm{~dB}$.

\section{SUMMARY}

It was shown that the MCF as defined for $\mathrm{Y}$-junctions, can also be used to describe the mode-splitting performance of a certain class of $\Psi$-junctions. Based on this a strategy was formulated for designing low crosstalk junctions. A mode-splitting $\Psi$-junction was fabricated using PECVD SiON 


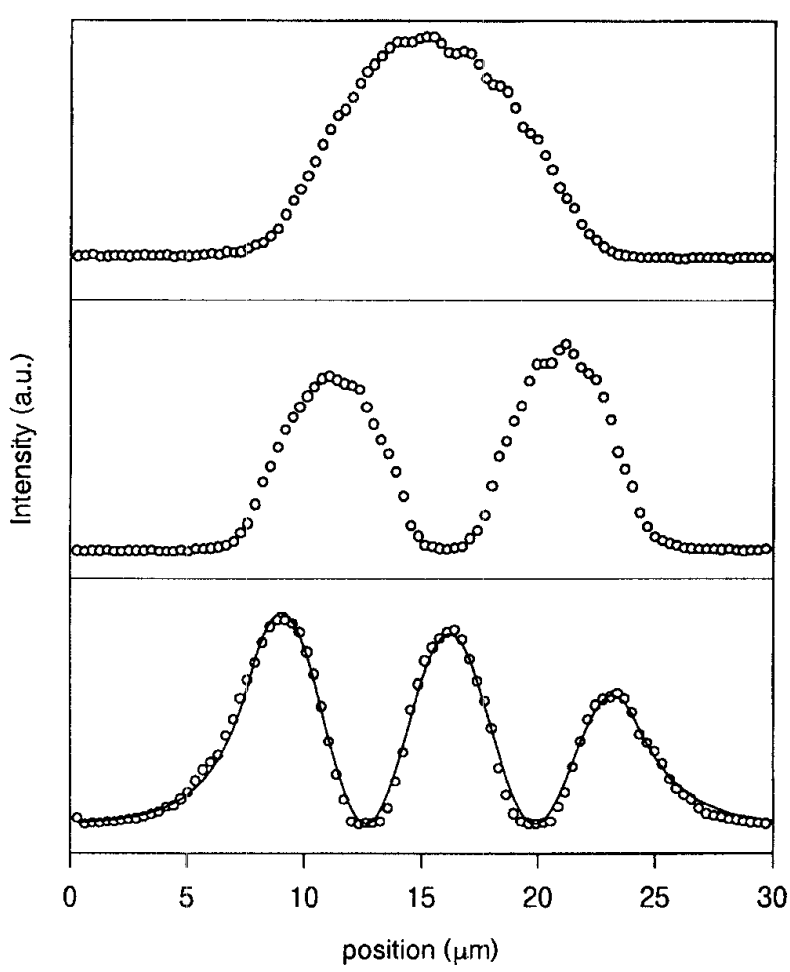

Fig. 7. Measured near-field mode profiles for exciting the different branches of a $\Psi$-junction. The line in the lower graph is a least-square fit on the measured data.

technology. Several characterization methods, e.g., analyzing power distributions over the branches, intensity profiles in the multimodal branch and the scattered beat-pattern, were used to determine the performance of this device. The propagation crosstalk, defined as the ratio between the power in the unwanted system modes and the total power in the system modes after propagation, was measured to be less than -17 $\mathrm{dB}$, the theoretical value being $-28 \mathrm{~dB}$. This difference can be attributed to inhomogeneities in the refractive indexes of both SiON layers.

\section{APPENDIX A}

In this appendix, we will show that equivalent $Y$-junctions [9] have the same MCF-value.

Berends et al. [9], showed that a normalized coordinate space can be defined for integrated optical devices, provided that only two refractive indexes are involved $\left(n_{g}\right.$ and $\left.n_{b}\right)$ and provided that the refractive index contrast is small (weakly guiding approximation). A device can be normalized in the $x$-(lateral) and $z$-(propagation) directions, using the following two transformations:

$$
\begin{aligned}
& \bar{x}=S_{x} x \\
& \bar{z}=S_{z} z
\end{aligned}
$$

with:

$$
\begin{aligned}
& S_{x}=k_{0} \sqrt{n_{g}^{2}-n_{b}^{2}} \\
& S_{z}=k_{0}\left(n_{g}-n_{b}\right)
\end{aligned}
$$

where $k_{0}$ is the free space wave vector.

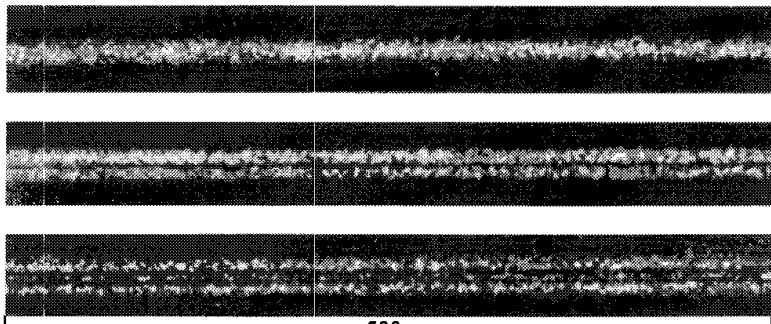

$500 \mu \mathrm{m}$

Fig. 8. Scatter pattern in the outgoing waveguide resulting from exciting the three different branches of a $\Psi$-junction.

With these scaling factors, a normalized angle $\theta_{n}$, for $\mathrm{Y}$ junctions can be defined as follows:

$$
\begin{aligned}
\tan \theta_{n} & \equiv \frac{\bar{x}}{\bar{z}} \\
& =\frac{S_{x} x}{S_{z} z} \\
& =\frac{S_{x}}{S_{z}} \tan \theta .
\end{aligned}
$$

Substituting the expressions for $S_{x}$ and $S_{z}$ we arrive at

$$
\tan \theta_{n}=\sqrt{\frac{n_{g}+n_{b}}{n_{g}-n_{b}}} \tan \theta .
$$

In the case of equivalent junctions, the normalized branching angle and the normalized refractive indexes [12] of the branches, $b_{1}$ and $b_{2}$, are equal. It can be shown that the MCF of a junction can be expressed in terms of these normalized pararneters only. The MCF can be written as

$$
\begin{aligned}
\mathrm{MCF} & =\frac{\Delta \beta}{\tan \theta \sqrt{\bar{\beta}^{2}-\beta_{b}^{2}}} \\
& =\frac{\Delta N}{\tan \theta \sqrt{\bar{N}^{2}-n_{b}^{2}}}
\end{aligned}
$$

with $\Delta N=N_{1}-N_{2}$ and $\bar{N}=\left(N_{1}+N_{2}\right) / 2$, where $N_{n}$ is the effective refractive index of branch $n$. In the weakly guiding approximation, $N$ can be written as [12]

$$
N=n_{b}+b\left(n_{g}-n_{b}\right) \text {. }
$$

Which allows us to write

$$
\Delta N=\Delta b\left(n_{g}-n_{b}\right)
$$

and

$$
\bar{N}=\bar{b}\left(n_{g}-n_{b}\right)+n_{b}
$$

where $\Delta b=b_{1}-b_{2}$ and $\bar{b}=\left(b_{1}+b_{2}\right) / 2$. By substitution, the MCF can now be rewritten as

$$
\mathrm{MCF}=\frac{\Delta b}{\sqrt{\bar{b}}} \cdot \frac{1}{\tan \theta} \cdot \sqrt{\frac{n_{g}-n_{b}}{\bar{b}\left(n_{g}-n_{b}\right)+2 n_{b}}} .
$$

Since $\left(n_{g}-n_{b}\right) \ll 2 n_{b}$, we can write in good approximation

$$
\bar{b}\left(n_{g}-n_{b}\right)+2 n_{b} \approx n_{g}+n_{b}
$$


and finally arrive at

$$
\begin{aligned}
\text { MCF } & =\frac{\Delta b}{\sqrt{\bar{b}}} \cdot \frac{1}{\tan \theta} \cdot \sqrt{\frac{n_{g}-n_{b}}{n_{g}+n_{b}}} \\
& =\frac{\Delta b}{\sqrt{\bar{b}}} \cdot \frac{1}{\tan \theta_{n}} .
\end{aligned}
$$

Since all variables in this expression are, by definition, the same for equivalent junctions, it has been proven that equivalent $\mathrm{Y}$-junctions have an identical MCF-value.

\section{APPENDIX B}

In the weakly guiding approximation, a measured near-field intensity distribution can be integrated in the vertical direction, after which the resulting one-dimensional mode profile $I(x)$ can be fitted with

$$
\begin{gathered}
I(x)=Y+\left[C_{0} \cdot E_{0}(x+X)+C_{1} \cdot E_{1}(x+X)\right. \\
\left.\cdot e^{j \phi_{1}}+C_{2} \cdot E_{2}(x+X) \cdot e^{j \phi_{2}}\right]^{2}
\end{gathered}
$$

in order to determine the power fraction in three different modes. Here $\phi_{1}$ and $\phi_{2}$ are the relative phases of the firstand the second-order mode, $C_{0}, C_{1}$, and $C_{2}$ are constants, $Y$ is the background intensity and $X$ is a constant that takes into account a lateral offset. $E_{0}(x), E_{1}(x)$, and $E_{2}(x)$ are the field profiles corresponding to the three lowest order TE slab modes as described by Kogelnik [12], which in normalized form are

$$
\begin{aligned}
& E_{i}(x) \propto \exp \left[-\sqrt{b_{i}} \cdot V \cdot\left(\frac{x}{w}-1\right)\right] \quad w<x \\
& \cos \left[\sqrt{1-b_{i}} \cdot V \cdot \frac{x}{w}-\arctan \sqrt{\left.\frac{b_{i}}{1-b_{i}}\right]}\right. \\
& E_{i}(x) \propto \frac{\sqrt{1-b}}{0<x<w} \\
& E_{i}(x) \propto \exp \left[\sqrt{b_{i}} \cdot V \cdot \frac{x}{w}\right] \quad x<0
\end{aligned}
$$

where $b_{i}$ is the normalized effective refractive index of mode $i, V$ is the normalized frequency of the waveguide and $w$ is the channel width. Together with the expression for the power carried by a mode

$$
P=\frac{\beta}{2 \omega \mu_{0}} \int_{x}|E(x)|^{2} d x
$$

where $w$ is the angular velocity of the light and $\mu_{0}$ is the magnetic permeability, the power fraction of the three different system modes in the measured near-field intensity distribution can be calculated.

\section{REFERENCES}

[1] H. Yajima, "Dielectric thin-film optical branching waveguide," Appl. Phys. Lett., vol. 22, pp. 647-649, 1973.

[2] E. Kapon and R. N. Thurston, "Multichannel waveguide junctions for guided-wave optics," Appl. Phys. Lett., vol. 50, pp. 1710-1712, 1987.

[3] A. Hardy, E. Marom, and Y. Shama, "Power transfer in asymmetric multiport couplers," Appl. Opt., vol. 27, pp. 447-448, 1988.

[4] R. N. Thurston, E. Kapon, and Y. Silberberg, "Analysis of mode separation in multichannel branching waveguides," IEEE J. Quantum Electron., vol. QE-23, pp. 1245-1255, 1987.

[5] W. K. Burns and A. F. Milton, "Mode conversion in planar-dielectric separating waveguides," IEEE J. Quantum Electron., vol. QE-11, pp. 32-39, 1975.

[6] G. J. M. Krijnen, H. J. W. M. Hoekstra, P. V. Lambeck, and T. J. M. A. Popma, "Simple analytical description of performance of Y-junctions," Electron. Lett., vol. 28, pp. 2072-2074, 1992.

[7] R. M. Knox and P. P. Toulios, in Proc. MRI Symp. Submillimeter Waves, J. Fox, Ed. Brooklyn, NY: Polytechnic Press, 1970.

[8] H. Yajima, "Coupled mode analysis of dielectric planar branching waveguides," IEEE J. Quantum Electron., vol. QE-14, pp. 749-755, 1978.

[9] J. H. Berends, G. J. Veldhuis, P. V. Lambeck, and Th. J. A Popma, "Device equivalence in integrated optics," J. Lightwave Technol., vol. 13, pp. 2082-2086, 1995.

[10] W. S. Bijlsma, H. Albers, P. V. Lambeck, and Th. J. A. Popma, "PECVD $\mathrm{SiO}_{r} \mathrm{~N}_{y}$ films as planar optical waveguides," in Sensors \& Actuators, Optical Transducers, A. Driessen, Ed. Deventer, The Netherlands: Kluwer Technical Books, 1990.

111] R. Ulrich and R. Torge, "Measurement of thin-film parameters with a prism coupler," Appl. Opt., vol. 12, pp. 2901-2908, 1973.

[12] H. Kogelnik, Topics in Applied Physics, T. Tamir, Ed. New York: Springer-Verlag, 1975 , vol. 7 , ch. 2.

G. J. Veldhuis was born in Nieuwolda, The Netherlands, in 1970. He recieved the M.Sc. degree in applied physics from the University of Twente, The Netherlands, in 1994 . He is currently pursuing the Ph.D. degree in the field of integrated optics for sensor applications.

J. H. Berends was born in Apeldoorn, The Netherlands, in 1969. He recieved the M.Sc. degree in electrical engineering from the University of Twente, The Netherlands, in 1992. He is currently persuing the Ph.D. degree, working on integrated optics narrowband filters.

P. V. Lambeck received the M.Sc. degree in physical chemistry from the University of Amsterdam in 1964. In the same year he joined the University of Twente, starting in the field of ferro-electric materials, a study which resulted in a Ph.D. dissertation.

Since 1984, he has been an Associate Professor in the Lightwave Device Group of the MESA Research Institute of the University of Twente, The Netherlands. His current interest is in the field of integrated optics, mainly on optical sensors and nonlinear devices. 